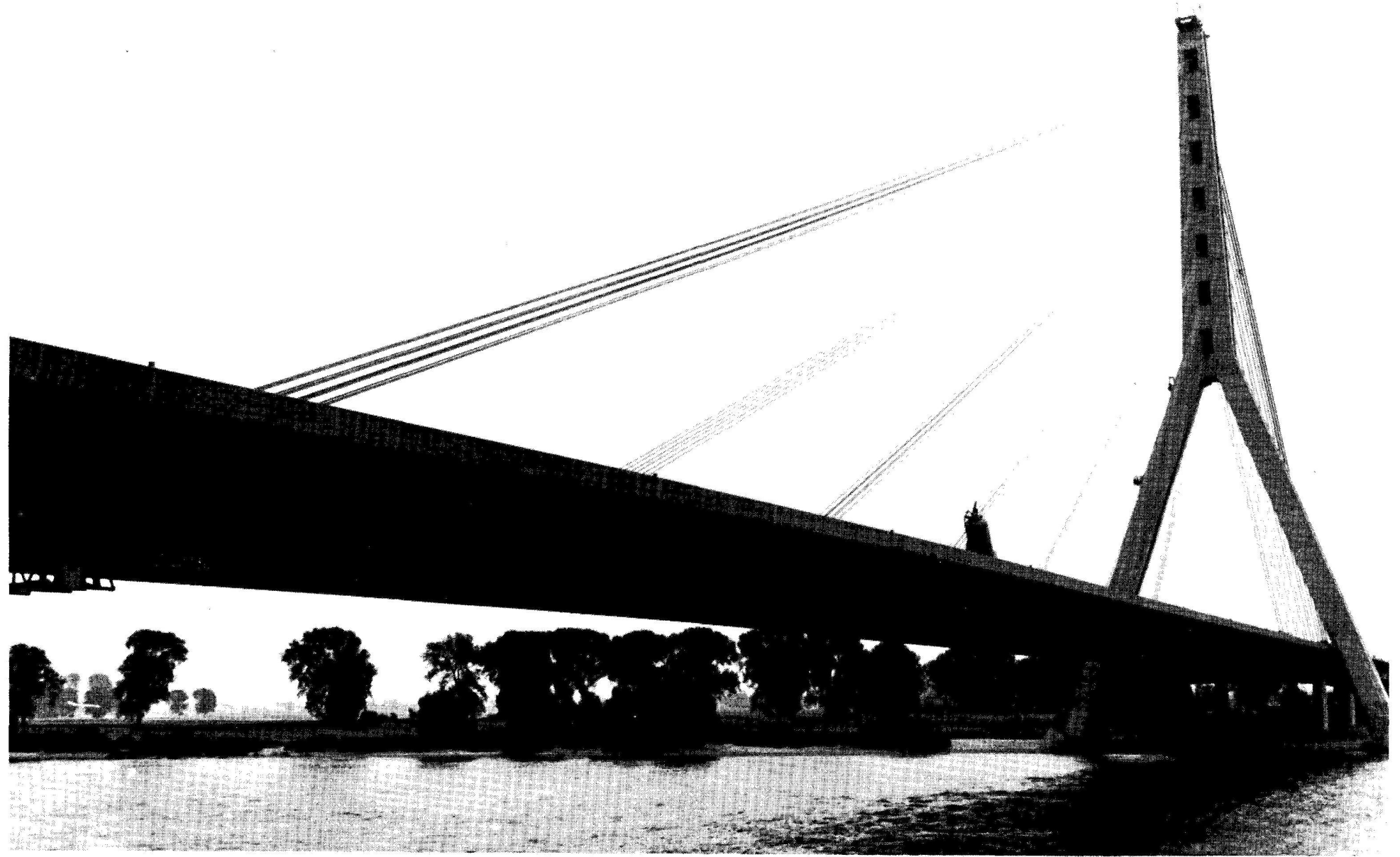

Fotos: SCHÄFER Y KRUPP RHEINHAUSEN

\title{
puente sobre el Rin
}

\section{sinopsis}

El puente sobre el Rin, en Düsseldorf-Flehe - una de cuyas funciones más importantes es la de aliviar el tráfico a los demás puentes existentes en la ciudad de Düsseldorf-, consta de tres partes claramente diferenciadas:

- una metálica de $368 \mathrm{~m}$ de longitud;

- otra de hormigón pretensado de $780 \mathrm{~m}$ de largo, y

- un pilono, en forma de A, que sirve como elemento de unión entre ambas.

Este puente ostenta el récord de luz libre en la República Federal Alemana, y su parte metálica constituye el voladizo de mayor longitud del mundo.

El puente de Düsseldorf-Flehe es una obra de $1.148 \mathrm{~m}$ de longitud total, que consta de tres partes claramente diferenciadas: un puente metálico atirantado de $368 \mathrm{~m}$ de largo, una zona de acceso de hormigón pretensado de $780 \mathrm{~m}$ de longitud y un pilono, en forma de $A$ prolongada en mástil superior, situado en la orilla derecha del río.

Los $368 \mathrm{~m}$ de puente metálico, cuyo dintel está formado por vigascajón de 16,3 m de ancho, constituyen la mayor luz libre existente en la República Federal Alemana, siendo a su vez el voladizo de mayor longitud del mundo.

\section{Düsseldorf Alemania Federal}

H. J. Modemann, Dipl. Ingeniero

564-33

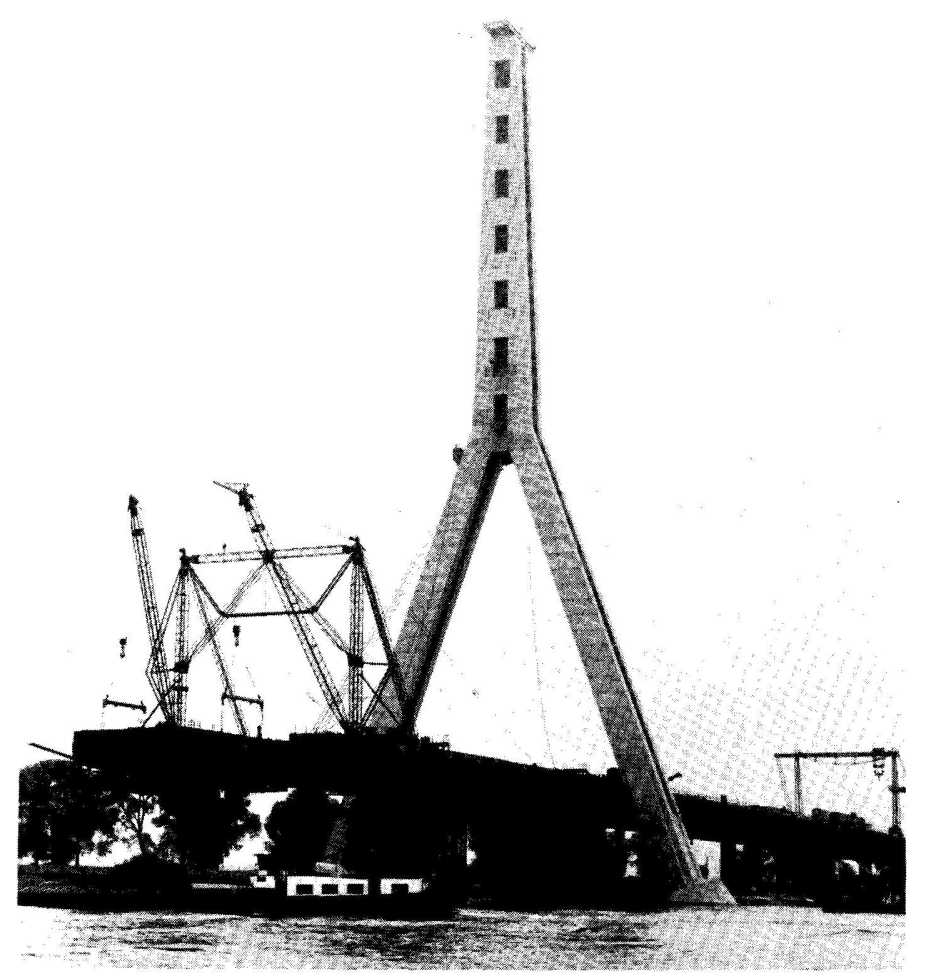

http://informesdelaconstruccion.revistas.csic.es 
Este puente de Flehe sirve para aliviar de tráfico a los otros cuatro puentes existentes en la capital del Estado (Süd, Knie, Uberkasseler y Theodor-Heuss) y comunica entre si varias autopistas, la A3 (Frankfurt-Oberhausen) con la A57 (Colonia-Krefeld) y la A59 (DüsseldorfLeverkussen) con la A46 (Grevenbroich-Wuppertal).

La concepción del puente se hizo pensando en un tráfico denso, por lo que se proyectaron tres carriles en cada sentido, un carril auxiliar y un paso combinado para peatones $y$ bicicletas.

El tablero del puente metálico, de una anchura total de $41,7 \mathrm{~m}$, es una placa anisotrópica ortogonal que sobresale $12,7 \mathrm{~m}$ a ambos lados de la viga-cajón y lleva unas vigas riostras transversales separadas $9 \mathrm{~m}$ entre sí.

El peso total del puente metálico, unas $7.000 \mathrm{t}$, es transmitido al mástil superior del pilono mediante siete cables de acero, los cuales anclan directamente en dicho mástil y se interrumpen al pasar por él. Estos cables, que tienen un diámetro que oscila entre 93 y 111 milímetros, soportan una fuerza de rotura de $13,4 \mathrm{MN}$ y pesan en total $1.200 \mathrm{t}$.

Los cálculos necesarios para el proyecto del puente se realizaron, primeramente, considerando la estructura de una manera total y, posteriormente, se estudiaron diversos sistemas parciales. En todos los cálculos se tuvo en cuenta la influencia de las deformaciones según el tamaño de la sección, el efecto de la retracción y de la fluencia de los elementos de hormigón sobre la estructura portante total y las diversas acciones de la temperatura.

En particular, se hicieron estudios detallados de la placa ortótropa de la calzada, del arriostramiento transversal, de los cables y de los pasadores de acero del pilono. El cálculo del pilono de hormigón pretensado, elemento de unión entre los puentes metálicos y de hormigón, se realizó según las últimas normas sobre hormigón pretensado.

Con los estudios realizados se eligió un pretensado adecuado para los cables, con lo que se consiguió una variación óptima de momentos bajo carga variable en el puente metálico y un estado libre de momentos bajo carga constante en el pilono.

En cuanto a los cables de montaje del voladizo del puente metálico, éstos requirieron un gran esfuerzo de cálculo para conseguir que los cables se pudiesen tensar ya definitivamente en el momento del montaje de tal modo que, al acabar éste, no hubiera necesidad de hacer ninguna conexión adicional.

Los trabajos de construcción de este puente, cuya empresa constructora principal fue la Krupp Industria de Rheinbussen, se iniciaron en marzo de 1976 y se abrió al tráfico en octubre de 1979.

\section{résumé}

PONT SUR LE RHIN - DUSSELDORF REPUBLIQUE FEDERALE D'ALLEMAGNE

J. Modemann, Ing. diplômé

Le pont sur le Rhin, à Düsseldorf-Fleche, dont l'une des fonctions les plus importantes est celle de rendre fluide le trafic des autres ponts existant à Düsseldorf, comprend trois parties distinctes:

- une partie métallique de $368 \mathrm{~m}$ de long, - une partie en béton précontraint de $780 \mathrm{~m}$ de long.

- un pylône, en $A$, qui sert d'élément d'union entre les deux parties précédentes.

Ce pont détient le record de portée libre dans la République fédérale d'Allemagne, et sa partie métallique constitue l'encorbellement le plus long du monde.

\section{summary}

BRIDGE OVER THE RHINE DUSSELDORF - WEST GERMANY

J. Modemann, Dipl. Engineer

The bridge over the Rhine, in DusseldorfFlehe - one of whose most important jobs is to alleviate traffic from the other bridges in the town of Dusseldorf - consists of three clearly diferentiated parts:

- a metal part $368 \mathrm{~m}$. in length

- another in prestressed concrete, $780 \mathrm{~m}$. long, and

- an A-shape pylon, acting as element joining both.

This bridge has the span clearance record in West Germany, and its metal part constitutes the longest corbel in the world.

\section{zusammenfassung}

RHEINBRUCKE - DUSSELDORF BUNDESREPUBLIK DEUTSCHLAND J. Modemann, Dipl. Ingenieur.

Die Rheinbrücke in Düsseldorf-Flehe, - unter deren wichtigsten Funktionen diejenige der Entlastung der übrigen in Düsseldorf vorhandenen Brücken zu nennen ist, besteht aus drei klar differenzierten Teilen:

- einen Metallteil von 368 m Länge;

- einen weiteren Spannbetonteil von $780 \mathrm{~m}$ Länge, und

- einen A-förmigen Pfeiler, der als Verbindungselement beider Teile dient.

Diese Brücke stellt den Rekord hinsichtlich freier Spannweite in der Bundesrepublik Deutschland dar, und ihr Metallteil ist der Jrösste freitragende Teil der Welt. 